\title{
Differential impact of ventromedial prefrontal cortex damage on "hot" and "cold" decisions under risk
}

\author{
Julia Spaniol $^{1} \cdot$ Francesco Di Muro $^{2} \cdot$ Elisa Ciaramelli ${ }^{2}$
}

Published online: 7 December 2018

(C) The Psychonomic Society, Inc. 2019

\begin{abstract}
The ventromedial prefrontal cortex (vmPFC) is known to play a key role in reward processing and decision making. However, its relative contribution to affect-rich ("hot") and affect-poor ("cold") decisions is not fully understood. Damage to vmPFC is associated with impaired performance on laboratory tasks of decision making under ambiguity and risk. In the current study, we tested the hypothesis that vmPFC is critical for adaptive risk taking under "hot" conditions specifically. Participants included patients with focal lesions in vmPFC, patient controls with damage in regions not including vmPFC, and healthy controls. They completed hot and cold versions of a dynamic risk-taking task, the Columbia Card Task (CCT). Relative to healthy controls and patient controls, vmPFC patients showed a strong overall increase in risk taking in the hot version of the CCT, despite preserved sensitivity to trial-level variation in risk. In the cold version, overall risk taking was similar among all three groups, even though vmPFC patients showed reduced sensitivity to trial-level variation in risk. Sensitivity to gain and loss magnitudes did not differ significantly among the groups, in either the hot or the cold CCT. These findings lend novel support to the hypothesis that the vmPFC is critical for adaptive decision making under affect-rich conditions.
\end{abstract}

Keywords Affect $\cdot$ Columbia Card Task $\cdot$ Decision making $\cdot$ Motivation $\cdot$ Reward $\cdot$ Risk taking

\section{Introduction}

As anyone who gambles frequently knows, it can be difficult to stop gambling "in the heat of the moment," following a streak of wins. The choice to walk away with one's winnings, or to take another gamble at the risk of a substantial loss, falls into the class of value-based decisions. These types of decisions require the integration of the subjective utilities of prospective gain and loss outcomes, as well as their respective probabilities (Rangel, Camerer, \& Montague, 2008). Disadvantageous value-based decision making is characteristic of multiple psychiatric conditions, including substance-use disorders and mood and anxiety disorders (Paulus, 2007). The

Electronic supplementary material The online version of this article (https://doi.org/10.3758/s13415-018-00680-1) contains supplementary material, which is available to authorized users.

Julia Spaniol

jspaniol@psych.ryerson.ca

1 Department of Psychology, Ryerson University, 350 Victoria Street, Toronto, ON M5B 2K3, Canada

2 Dipartimento di Psicologia, Università di Bologna, Bologna, Italy ventromedial prefrontal cortex (vmPFC), an area that includes the medial orbitofrontal cortex and the ventral aspects of the medial prefrontal cortices, is known to play a role in valuation and risk assessment, as well as in affective function more generally (Roy, Shohamy, \& Wager, 2012). In the current study we used a lesion approach to test the hypothesis that vmPFC is critical for adaptive value-based decisions under affect-rich, "hot" conditions.

\section{Value and the vmPFC}

FMRI studies of reward processing in healthy individuals consistently show BOLD activity in vmPFC associated with the anticipation and receipt of reward and punishment (Metereau \& Dreher, 2015; O'Doherty, Kringelbach, Rolls, Hornak, \& Andrews, 2001). The observation that vmPFC activity is sensitive to different reward types (e.g., money, food), across a variety of choice tasks, has led to the proposal that vmPFC represents a "common currency" of subjective value (Levy \& Glimcher, 2012). Recent fMRI evidence suggests that vmPFC not only responds to momentary gains and losses, but also tracks cumulative reward histories (Juechems, Balaguer, Ruz, \& Summerfield, 2017) that are accompanied by shifts in risk preference. Support for the idea that vmPFC 
plays a role in the construction of subjective utility has also come from lesion studies in which patients with focal vmPFC damage were compared to patients with damage to other brain regions, as well as to healthy controls. While habitual valuebased choice appears intact, goal-directed choice has been shown to be impaired in vmPFC patients (Reber, Feinstein, O'Doherty, Liljeholm, Adolphs, \& Tranel, 2017). vmPFC may also play a role in the fidelity with which values are assigned to options. In a task requiring simple preference judgments between pairs of same-category objects (e.g., an apple vs. a banana), vmPFC patients made more erratic preference judgments, committing more frequent violations of transitivity (e.g., $\mathrm{A}>\mathrm{B}$ and $\mathrm{B}>\mathrm{C}$, but $\mathrm{C}>\mathrm{A}$; HenriBhargava, Simioni, \& Fellows, 2012) compared with control participants. Overall, this literature suggests that vmPFC plays a causal role in valuation and goal-directed control, which in turn are critical for effective value-based decision making.

\section{Risk and the vmPFC}

Seminal early studies on risk taking and the vmPFC examined the effects of vmPFC damage on performance in the Iowa Gambling Task (Bechara, Damasio, Damasio, \& Anderson, 1994; Bechara, Damasio, Tranel, \& Damasio, 1997; Bechara, Tranel, Damasio, \& Damasio, 1996). The Iowa Gambling Task requires participants to learn, via trial and error, which of four decks of cards are associated with advantageous long-term outcomes. As such, the Iowa Gambling Task involves decisions under ambiguity, with successful performance taxing learning and memory. Patients with vmPFC damage fail to learn to select the advantageous decks, and do not show anticipatory skin conductance responses in advance of selections of the disadvantageous decks. More recent lesion studies have employed a variety of risky-choice tasks designed to isolate specific decision processes, separate from learning and memory, that are affected by vmPFC damage.

In the Cambridge Gamble Task, outcome probabilities are explicitly provided, minimizing the contribution of memory on decision making. Participants are presented with a set of ten red and blue boxes, one of which contains a hidden token. The ratio of red to blue varies from trial to trial. After guessing the color of the box under which the token is most likely hidden, participants are asked to indicate how much they are willing to bet on their guess. Each decision is followed by immediate feedback regarding the number of points won or lost. In a study comparing patients with damage to vmPFC, patients with damage to insular cortex, and healthy controls in the Cambridge Gamble Task (Clark et al., 2008), different deficits emerged in the two patient groups. Relative to healthy controls, both patient groups made higher bets overall. Whereas vmPFC patients resembled healthy controls with respect to their risk adjustment (i.e., sensitivity of betting to triallevel differences in the probability of winning, defined by the ratio of red to blue boxes), insula patients failed to demonstrate risk adjustment.

The Roulette Betting Task (Studer \& Clark, 2011) is similar to the Cambridge Gamble Task, in that outcome probabilities are explicitly provided and participants are asked to place bets on these outcomes. However, in the Roulette Betting Task, the probability of winning ranges from high to low, producing a wider range of optimal bets than the Cambridge Gamble Task. Studer, Manes, Humphreys, Robbins, and Clark (2015) compared patients with damage to vmPFC, patients with damage to posterior parietal cortex, and healthy controls on the Roulette Betting Task. Both patient groups showed impaired risk adjustment relative to healthy controls, and vmPFC patients additionally demonstrated increased risk appetite. Interestingly, both patient groups performed normally on control tasks that involved probabilistic reasoning in the absence of risky choice.

A disadvantage of the Cambridge Gamble Task and the Roulette Betting Tasks is that neither yields separate indices of gain and loss sensitivity. Therefore, these tasks do not lend insight into potential interactions of the effects of vmPFC damage and domain (gain vs. loss) on risk taking. Weller, Levin, Shiv, and Bechara (2007) used a risky-choice paradigm (the "cups task") with explicit outcome probabilities that presents gain- and loss-related decision scenarios in separate task trials. On each trial of the task, participants choose between a "sure thing" option and a risky option. No trial-level feedback is provided. VmPFC patients exhibited impaired risk adjustment on both gain and loss trials, whereas patients with damage to the amygdala showed lack of risk adjustment on gain trials only.

A more recent study (Pujara, Wolf, Baskaya, \& Koenigs, 2015) used a financial decision-making task similar to those employed in Kahneman and Tversky's (1979) classic work on risky choice. Each trial involved a choice between a sure outcome (e.g., "lose \$40") and a gamble (e.g., "50\% chance of losing $\$ 50$, else lose $\$ 0 "$ ). No post-decision feedback was given. The study included patients with focal vmPFC damage, control patients with damage outside the vmPFC, and healthy controls. All groups demonstrated risk avoidance for gains and risk seeking for losses. However, this pattern - known as the reflection effect (Kahneman \& Tversky, 1979) - was more pronounced among vmPFC patients than in the control groups. The authors interpreted this finding in terms of a role of vmPFC in triggering affective responses to risky options. By this account, decisions are dominated by affective responses to non-risky options (sure gain, sure loss), thus producing the reflection effect. However, in Pujara et al.'s (2015) study, all decisions involved hypothetical risks and rewards. It cannot be ruled out that the increased reflection effect among vmPFC patients may have resulted from the computational demands of comparing hypothetical sure and risky options. 
In summary, there is evidence for a causal role of vmPFC in adaptive risk taking in the face of gains and losses, both in terms of excessive risk appetite and lack of risk adjustment. To what extent affective engagement plays a role in these deficits is still unclear.

\section{Affective meaning and the vmPFC}

Valuation and decision making are not the only functions in which vmPFC has been implicated. Other examples include self-directed cognition (D'Argembeau, 2013), memory and prospection (Bertossi, Tesini, Cappelli, \& Ciaramelli, 2016), mind-wandering (Bertossi \& Ciaramelli, 2016), and emotion (Myers-Schulz \& Koenigs, 2012). According to one recent proposal (Roy et al., 2012), the common theme connecting these functions is the generation of affective meaning. By this account, vmPFC is a neural hub that supports the construction of context-sensitive emotional responses by integrating information about current internal and external states with relevant representations from long-term memory. In this view, vmPFC should play a critical role in supporting adaptive decision making under conditions in which affective meaning must be continuously updated on the basis of dynamic situational cues.

\section{The current study}

The extant literature on the role of $\mathrm{vmPFC}$ in risky choice suggests that vmPFC is critical for regulating risk appetite, and that it may also play a role in facilitating risk adjustment (i.e., calibration of risk taking as a function of variation in risk). In line with the proposal that vmPFC is a hub that constructs affective meaning from internal and external stimuli to facilitate adaptive behavior (Roy et al., 2012), we hypothesize that damage to vmPFC produces decision-making deficits particularly under "hot" conditions that are characterized by dynamically shifting situational contexts. This hypothesis is also informed by the observation, in a recent fMRI study (Juechems et al., 2017), that vmPFC tracks cumulative reward, thereby facilitating increases in risk aversion with increasing winnings.

We used the Columbia Card Task (CCT; Figner et al., 2009; Figner \& Weber, 2011) to compare vmPFC patients to patients with damage in brain regions not including vmPFC, as well as healthy controls. The CCT is a risky choice task in which three decision-relevant parameters are manipulated factorially across task trials: gain magnitude, loss magnitude, and risk (defined as the probability of a loss). Critically, the task provides the opportunity to compare decision performance under "hot" and "cold" conditions. The hot CCT involves incremental choices with immediate feedback. It engages affective processes and elicits greater physiological arousal compared to the cold CCT (Figner et al., 2009;
Holper \& Murphy, 2014). The cold CCT, in contrast, gives rise to a more deliberative decision strategy. The CCT has been shown to demonstrate both individual and developmental differences (Figner et al., 2009; Figner \& Weber, 2011; Panno, Lauriola, \& Figner, 2013), but to our knowledge, it has not been used in prior lesion studies.

Using the CCT, we sought to examine risk appetite and risk adjustment under both hot and cold conditions. We predicted that differences between the vmPFC group and the control groups would be more pronounced in the hot CCT than in the cold CCT, with respect to both overall risk appetite and risk adjustment. In addition to performance on the CCT, we also examined cognitive and affective measures that could potentially influence risky choice, including fluid intelligence, numeracy, and mood.

\section{Method}

\section{Participants}

Participants included 12 patients with brain damage and 30 healthy individuals (see Table 1 for demographic and clinical characteristics). Patients were recruited at the Centre for Studies and Research in Cognitive Neuroscience, Cesena, Italy.

Patients were selected on the basis of the location of their lesion evident on MRI or CT scans. Six patients had lesions involving the vmPFC (vmPFC patients) as a result of the rupture of an aneurysm of the anterior communicating artery $(\mathrm{ACOA})$. VmPFC lesions were bilateral in all cases, though in many cases asymmetrical. Lesion analysis, performed with MRIcro (Rorden \& Brett, 2000), showed that the Brodmann areas (BAs) mainly affected included BAs 10, 11, 24, 25, and 32, with the region of maximal overlap located in BA $11(M=19.19 \mathrm{cc}, S D=$ 12.82), BA $10(M=12.04 \mathrm{cc}, S D=7.35)$, and BA $32(M=$ $7.88 \mathrm{cc}, S D=5.15$; see Fig. 1). In one patient, the lesion extended to the dorsolateral prefrontal cortex (BA 9, accounting for $8 \%$ of total lesion volume, and BA 46, accounting for $9 \%$ of total lesion volume) and ventrolateral prefrontal cortex (BA 47, accounting for $4 \%$ of total lesion volume). Considering that the size of this patient's medial prefrontal lesion was almost triple that of his lateral prefrontal lesion, we decided to include him in the patient sample. Excluding this patient from the analyses did not alter the pattern of results.

Six patients had lesions not involving vmPFC (control patients). In this group, lesions were unilateral in five cases (two in the left hemisphere) and bilateral in one case, and were caused by stroke (four cases) or brain tumor (two cases). Lesion sites mainly included the occipital and occipitotemporal cortex (BAs 17, 18, 19, and 37; six cases). One 
Table 1 Demographic, cognitive, and affective characteristics

\begin{tabular}{|c|c|c|c|c|c|c|c|c|c|}
\hline Patient & Sex & Age (years) & Edu (years) & $\begin{array}{l}\text { Time since } \\
\text { lesion (months) }\end{array}$ & MMSE & RSPM & ANS & PANAS-POS & PANAS-NEG \\
\hline \multicolumn{10}{|c|}{ vmPFC patients } \\
\hline 1 & M & 55 & 8 & 60 & 30 & 32 & 6 & 31.5 & 23 \\
\hline 2 & M & 45 & 13 & 60 & 26 & 33 & 10 & 40.5 & 15 \\
\hline 3 & M & 60 & 13 & 168 & 26 & 18 & 7 & 14 & 10 \\
\hline 4 & M & 55 & 8 & 132 & 24 & 34 & 9 & 23.5 & 12 \\
\hline 5 & M & 53 & 13 & 24 & 28 & 27 & 7 & 30.5 & 11 \\
\hline 6 & $\mathrm{~F}$ & 53 & 11 & 12 & 28 & 30 & 9 & 33 & 10 \\
\hline$M(S D)$ & & $53.50(4.89)$ & $11.00(2.45)$ & $76.00(61.50)$ & $27.05(2.07)$ & $28.75(5.84)$ & $8.00(1.55)$ & $28.83(9.08)$ & $13.50(5.01)$ \\
\hline \multicolumn{10}{|c|}{ Patient controls } \\
\hline 7 & M & 36 & 14 & 8 & 28 & 32 & 11 & 34.5 & 11 \\
\hline 8 & M & 75 & 18 & 8 & 29 & 36 & 9 & 26 & 10 \\
\hline 9 & M & 59 & 8 & 45 & 30 & 31 & 10 & 36 & 11 \\
\hline 10 & $\mathrm{~F}$ & 33 & 16 & 6 & 28 & 32 & 11 & 40 & 14.5 \\
\hline 11 & M & 65 & 13 & 8 & 29 & 41 & 9 & 33.5 & 10 \\
\hline 12 & $\mathrm{~F}$ & 67 & 5 & 10 & 27 & 31 & 6 & 33 & 13.5 \\
\hline$M(S D)$ & & $55.83(17.33)$ & $12.33(4.93)$ & $14.17(15.16)$ & $28.54(1.16)$ & $33.88(3.97)$ & $9.33(1.86)$ & $33.83(4.59)$ & $11.67(1.89)$ \\
\hline \multicolumn{10}{|c|}{ Healthy controls } \\
\hline$M(S D)$ & - & $52.53(5.23)$ & $10.90(2.26)$ & - & $28.66(.87)$ & $29.02(6.00)$ & $8.23(2.39)$ & $34.27(7.16)$ & $12.27(2.81)$ \\
\hline
\end{tabular}

$M$ male, $F$ female, Edu education, $v m P F C$ ventromedial prefrontal cortex, MMSE Mini-Mental State Examination, $R S P M$ Raven's Standard Progressive Matrices, ANS Abbreviated Numeracy Scale, PANAS-POS positive affect score, Positive Affect and Negative Affect Schedule (averaged across Sessions 1 and 2), PANAS-NEG negative affect score, Positive Affect and Negative Affect Schedule (averaged across Sessions 1 and 2).

Age range for healthy controls: $41-60$ years. Education range for healthy controls: 8-13 years

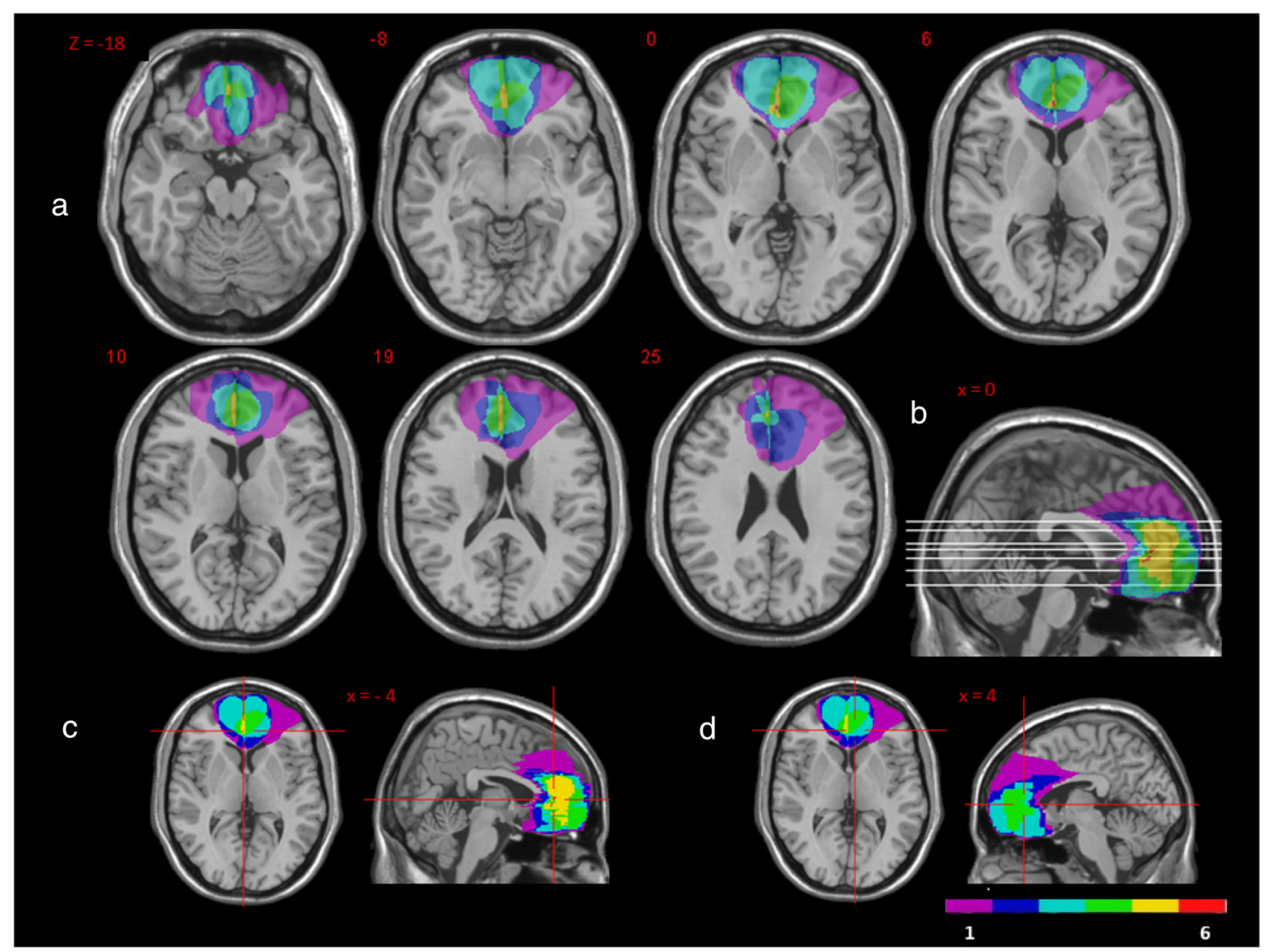

Fig. 1 Location and overlap of $\mathrm{vmPFC}$ lesions. The figure shows the lesions of the six patients with vmPFC damage projected on the same six axial slices (A), on the midline cumulative sagittal view of the standard Montreal Neurological Institute brain (B), and on lateral sagittal slices from the left $(\mathbf{C})$ and right $(\mathbf{D})$ hemisphere. The left hemisphere is shown on the left side. The color bar indicates the number of overlapping lesions (1-6). Maximal overlap is present in Brodmann areas 10,11 , and 32, mainly in the left hemisphere 
additional control patient failed to return for the second session of testing and was not included in the final dataset. There was no significant difference in lesion volume between vmPFC patients and control patients (54.34 vs. $22.29 \mathrm{cc}$, Mann-Whitney $U=9, z=1.44, p=.15$ ).

All patients were tested at least six months after the onset of their brain injury (vmPFC patients: median $=60$, range $=12-$ 168; patient controls: median $=8$, range $=6-45$; see Table 1 ). They were not taking psychoactive drugs and had no other diagnosis likely to affect cognition (e.g., significant psychiatric disease or alcohol abuse).

The healthy control group included 30 individuals (five female, 25 male). They were screened for use of psychoactive drugs, alcohol and drug abuse, epilepsy, and any other known neurological or psychiatric conditions. Seven additional control participants were tested but were later excluded because they reported suspecting that the computer task was rigged to prevent loss outcomes (three participants), because they completed both sessions in a single day, instead of over a span of two or more days, due to scheduling errors (three participants), or because of difficulties using the computer (one participant). Across the three participant groups, one-way analyses of variance (ANOVAs) showed no significant differences in age, $F(2,39)=.45, p=.64, \eta_{\mathrm{p}}{ }^{2}=.02$, or education, $F(2,39)=$ $.67, p=.52, \eta_{\mathrm{p}}{ }^{2}=.03$. All participants gave informed consent, and all study procedures were approved by the Bioethical Committee of the University of Bologna, and the CEIIAV Ethical Committee of Emilia Romagna Regional Health Service.

\section{Cognitive and affective profile}

Table 1 shows scores on standard tests of cognitive and affective function. Patients scored above cutoff on the Mini Mental State Examination (MMSE; Folstein et al., 1975), a screening measure for dementia, and performed in the normal range on Raven's Standard Progressive Matrices (RSPM; Spinnler \& Tognoni, 1987). A one-way ANOVA on RSPM scores with participant group as the between-subjects factor showed no group differences, $F(2,39)=1.87, p=.17, \eta_{\mathrm{p}}{ }^{2}=.09$. In contrast, scores on the MMSE showed a significant group effect, $F(2,39)=5.05, p=.01, \eta_{\mathrm{p}}{ }^{2}=.21$. Bonferroni post hoc comparisons indicated that MMSE scores were lower in vmPFC patients compared to healthy controls, $p<.01$, but were not significantly different from those of patient controls, $p=.09$.

Participants also completed the Abbreviated Numeracy Scale (ANS; Weller et al., 2013), which includes 11 items assessing basic probability reasoning abilities (e.g., "Imagine that we roll a fair, six-sided dice 1,000 times. Out of 1,000 rolls, how many times do you think the die would come up even: 2,4 , or 6?"). There were no significant group differences in ANS scores, $F(2,39)=.70, p=.51, \eta_{\mathrm{p}}{ }^{2}=.03$.
Affective states were assessed using the Positive and Negative Affect Schedule (PANAS; Terracciano, McCrae, \& Costa, 2003; Watson, Clark, \& Tellegen, 1988), a self-report scale requiring participants to rate the extent to which they were currently experiencing each of 20 emotions (ten positive, ten negative) on a 5-point Likert scale ranging from "very slightly" to "very much." Participants completed the scale twice, once in each experimental session. A mixed ANOVA on positive mood scores, with the between-subjects factor group and the within-subjects factor session, yielded no significant effects of group, $F(2,39)=1.45, p=.25, \eta_{\mathrm{p}}{ }^{2}=.07$, session, $F(1,39)=.02, p=.90, \eta_{\mathrm{p}}{ }^{2}<.00$, or their interaction, $F(2,39)=1.51, p=.22, \eta_{\mathrm{p}}{ }^{2}=.07$. Likewise, a mixed ANOVA on negative mood scores showed no significant effects of group, $F(2,39)=.57, p=.57, \eta_{\mathrm{p}}{ }^{2}=.03$, session, $F(1,39)=$ $1.00, p=.76, \eta_{\mathrm{p}}{ }^{2}<.00$, or their interaction, $F(2,39)=.91, p=$ $.41, \eta_{\mathrm{p}}^{2}=.05$.

\section{Columbia card task}

In both the hot and cold versions of the CCT (Figner et al., 2009; see Fig. 2), participants see 32 cards arranged in four rows of eight cards. The objective is to accumulate points by turning over cards. Most of the 32 cards in the set are "gain cards," and a small subset are "loss cards." The participant can turn over cards and gain points until a loss card is encountered, at which point the trial ends and the loss is deducted from the running total. All cards are initially shown face down, making it impossible to tell which of the cards are loss cards. At the top of the computer screen, three values are displayed that provide information about the loss amount (i.e., the number of points deducted if a loss card is turned over), the gain amount (i.e., the number of points added if a gain card is turned over), and the number of loss cards hidden in the set of 32 cards, which determines the probability of a loss. The number of the current trial is also shown in the upper left corner of the screen. The CCT yields two measures: overall risk taking or risk appetite (Studer et al., 2015), defined as the average number of cards turned over, and information use (Figner et al., 2009) or risk adjustment (Studer et al., 2015), defined as the extent to which participants' risk taking is sensitive to the three parameters of loss amount, gain amount, and loss probability.

In the hot CCT, participants turn over cards by clicking on them using the computer mouse. Each click results in immediate feedback, revealing the front side of the card. For gain cards, the front side shows a yellow happy face icon, whereas for loss cards, the front side shows a red sad face icon. Furthermore, with each gain card that is turned over, the cumulative point total, shown in the top-right corner of the screen, increases by the current gain amount. If a loss card is turned over, the trial terminates, and the loss amount is subtracted from the cumulative point total. The participant 

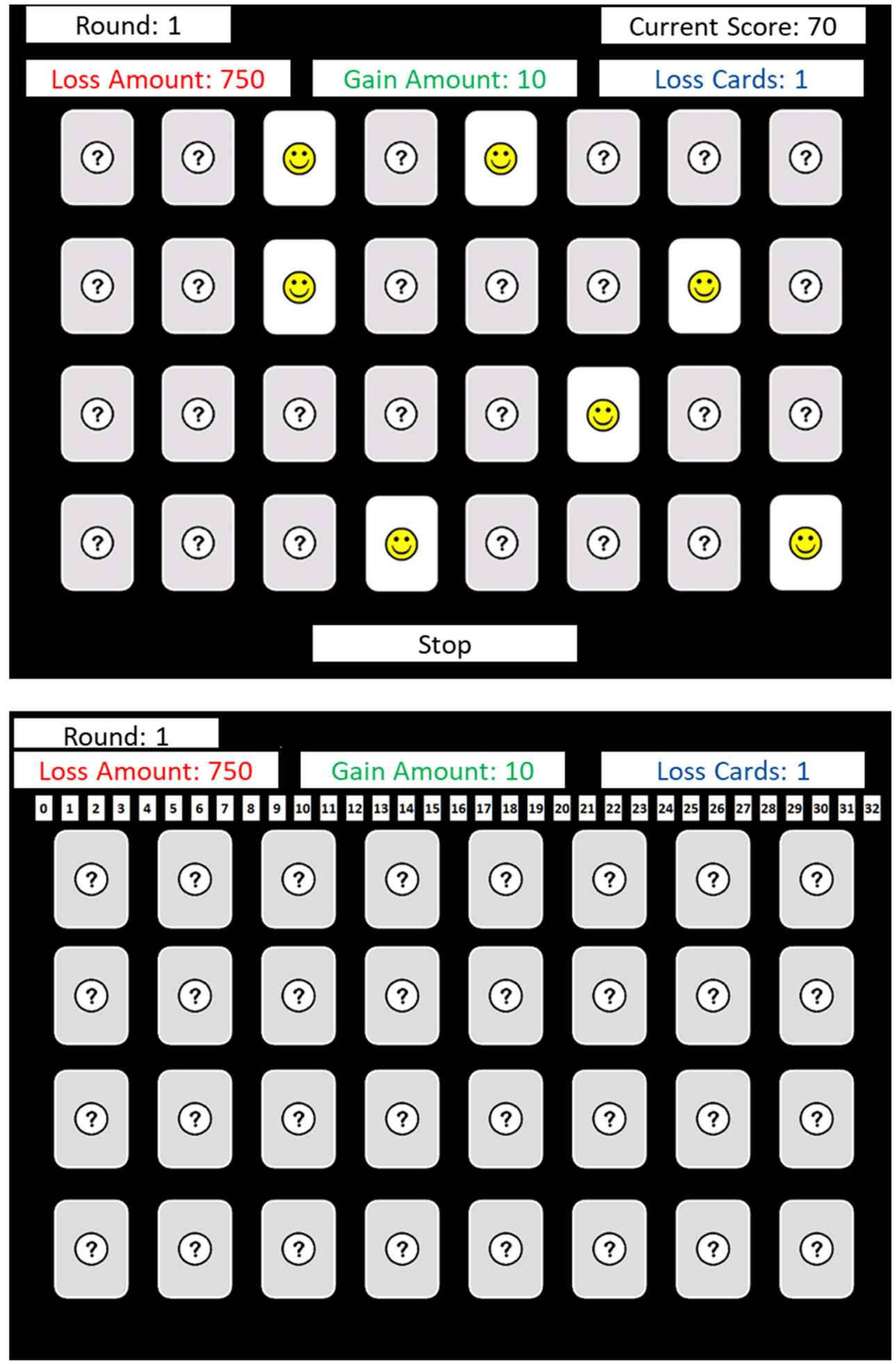

Fig. 2 Illustration of the hot (top panel) and cold (bottom panel) Columbia Card Task

can stop the trial at any point by clicking the "Stop" button at the bottom of the screen, thereby initiating the onset of the next trial. The hot CCT thus presents a dynamic risk-taking context in which every incremental decision (to turn over a card, or to terminate the trial) results in immediate feedback.
The number of cards turned over on a given trial is an informative measure of risk taking only if the participant has stopped the trial voluntarily. If the trial ends because the participant has encountered a loss card, it is possible that the participant would have continued to turn over cards if not 
for the involuntary termination. For this reason, the hot CCT is rigged so that participants never encounter a loss card until they have turned over almost all of the 32 cards in the set (e.g., on a trial with three loss cards, the 30th card turned over is a loss card). To reduce the likelihood that participants become aware of the rigged nature of the task, 12 dummy trials are included in which loss cards appear within the first ten selections.

The cold CCT involves no immediate feedback. Instead of turning over individual cards, participants make a single decision about how many cards to turn over in a given trial. They click the corresponding number box above the card array and continue on to the next trial. No feedback about cumulative point totals is provided until the end of the task. It is important to note that the structure of the task is identical for the cold and the hot versions, including the presence of information about loss probability, loss amount, and gain amount, the inclusion of dummy trials, and the fact that if a loss card is selected the loss amount for that card is subtracted from the payoff. However, in the cold version, participants receive no feedback during the task and remain unaware of gain and loss outcomes on individual trials.

\section{Design}

The study employed a full factorial design with the betweensubjects variable group (vmPFC patient, patient control, healthy control) and four within-subject variables: (a) CCT version (hot vs. cold), administered in separate experimental sessions, (b) the loss probability (1, 2, or 3 loss cards), (c) the gain amount $(10,20$, or 30 points per gain card), and (d) the loss amount (250, 500, or 750 points). Within each session, the 27 unique combinations of the levels of loss probability, gain amount, and loss amount were each presented twice, for a total of 54 experimental trials per session. Within each block of 27 trials, trial order was random. The 12 additional dummy trials were randomly intermixed with the experimental trials in each session, yielding 66 trials/session in total. Unless otherwise stated, dummy trials were not included in any of the analyses reported below.

\section{Procedure}

All participants completed both the hot and cold versions of the CCT (Figner et al., 2009). The two versions were administered on different days, with version order counterbalanced across participants within each group. Both sessions were administered individually by the same researcher, in a quiet room. In Session 1, participants completed the consent form, a demographic and health questionnaire, and the PANAS (Terracciano et al., 2003; Watson et al., 1988). The researcher then presented participants with the instructions for the hot or cold CCT, depending on the counterbalancing condition. The instructions included a series of written explanations and pictorial illustrations that were presented on the computer screen. Before starting the task, participants had to pass a comprehension check consisting of four questions. The questions probed the participant's understanding of the task, including the consequences of drawing gain and loss cards, and the payout scheme. If the participant gave incorrect answers to any of the questions, the researcher provided additional clarification until the participant demonstrated complete understanding of the rules. The participant then completed the CCT at his or her own pace, and received a small payout once finished. Participants were told that the payout was a function of their points accumulated on a single, randomly-selected trial of the CCT, but in fact the payout was drawn randomly from the $€ 3.00-5.00$ range to prevent large discrepancies in individual payments that may have affected participants' motivation in Session 2. Participants then completed the RSPM (Spinnler \& Tognoni, 1987) and the MMSE (Folstein et al., 1975).

Session 2 took place at least 1 day after Session 1 (median inter-session lag $=2.5$ days). Participants completed the PANAS and received task instructions for the CCT (hot or cold, depending on the counterbalancing condition). They then completed the CCT at their own pace and received their Session 2 payout. After completion of the CCT, the researcher asked participants whether they had noticed anything about the task. This was done to identify individuals who had suspected that the CCT was rigged. After this, participants completed the ANS (Weller et al., 2013) and received a full debriefing about the purpose of the study.

\section{Results}

The number of cards turned over, an index of risk taking in the CCT, served as the primary dependent measure. Descriptive statistics for the number of cards turned over, calculated separately for each group and experimental condition, are provided in the Online Supplementary Material. We analyzed these data with a mixed ANOVA, with group (vmPFC patients, control patients, healthy controls) as a between-subjects factor and version (hot, cold), loss probability (1, 2, or 3 loss cards), gain amount $(10,20$, or 30 points), and loss amount $(250,500$, or 750 points) as within-subjects factors. Significant main effects and interactions were followed up with Bonferronicorrected post hoc tests. In light of the small sample size in both patient groups, we additionally report non-parametric post hoc tests to follow up significant ANOVA effects that involved the group factor.

For ease of interpretation, we break down the ANOVA results into two sets. We first report the effects characterizing group and version effects on the number of cards turned over, regardless of the task parameters that varied from trial to trial (gain amount, loss amount, and loss probability). These 
effects provide a picture of overall risk appetite (Studer et al., 2015). Next we report the effects of information use (Figner et al., 2009), that is, sensitivity to trial-level variations in gain amount, loss amount, and loss probability. We tested only the main effects of these within-subjects factors, as well as their interactions involving the group factor, since these were the only effects relevant to the current research questions.

Risk appetite The main effect of group on risk taking was significant, $F(2,39)=6.02, p<.01, \eta_{\mathrm{p}}{ }^{2}=.24$, as was the main effect of version, $F(1,39)=29.86, p<.01, \eta_{\mathrm{p}}{ }^{2}=.43$, and the Group $\times$ Version interaction, $F(2,39)=15.90, p<.01, \eta_{\mathrm{p}}{ }^{2}=$ .45. Bonferroni follow-up comparisons revealed that healthy controls and patient controls did not differ significantly from each other, $p=.19$, but vmPFC patients differed significantly from each of the other groups, $p<.01$, showing increased risk taking (i.e., turning over more cards). A second set of Bonferroni follow-up tests examined version effects (hot vs. cold) on risk taking within each participant group. There was no significant effect for patient controls, $p=.23$ or for healthy controls, $p=.13$. For vmPFC patients, however, the version effect was significant, $p<.01: \mathrm{VmPFC}$ patients turned over more than twice as many cards in the hot version than in the cold version. These results are illustrated in Fig. 3 (top panel).

Given the small sample sizes in the patient groups, we sought to corroborate the results of the post hoc comparisons with nonparametric tests. A Kruskal-Wallis test yielded no significant group effect in the cold version, $\chi^{2}(2)=2.34, p=$ .31 , but a significant effect of group emerged in the hot version, $\chi^{2}(2)=13.03, p<.01$. Pairwise follow-up comparisons using the Mann-Whitney procedure for independent samples revealed that healthy controls and patient controls did not differ significantly from each other, $U=49, z=1.74, p=$ .08. However, vmPFC patients differed significantly from healthy controls, $U=15.00, z=3.18, p<.01$, and from patient controls, $U=2.00, z=2.56, p=.01$, showing increased risk taking (i.e., turning over more cards). A second set of nonparametric post hoc tests, using the Wilcoxon signed-rank procedure for related samples, examined version effects (hot vs. cold) on risk taking within each participant group. There was no significant effect for healthy controls, $z=1.21, p=.23$, or for patient controls, $z=1.15, p=.25$. For vmPFC patients,
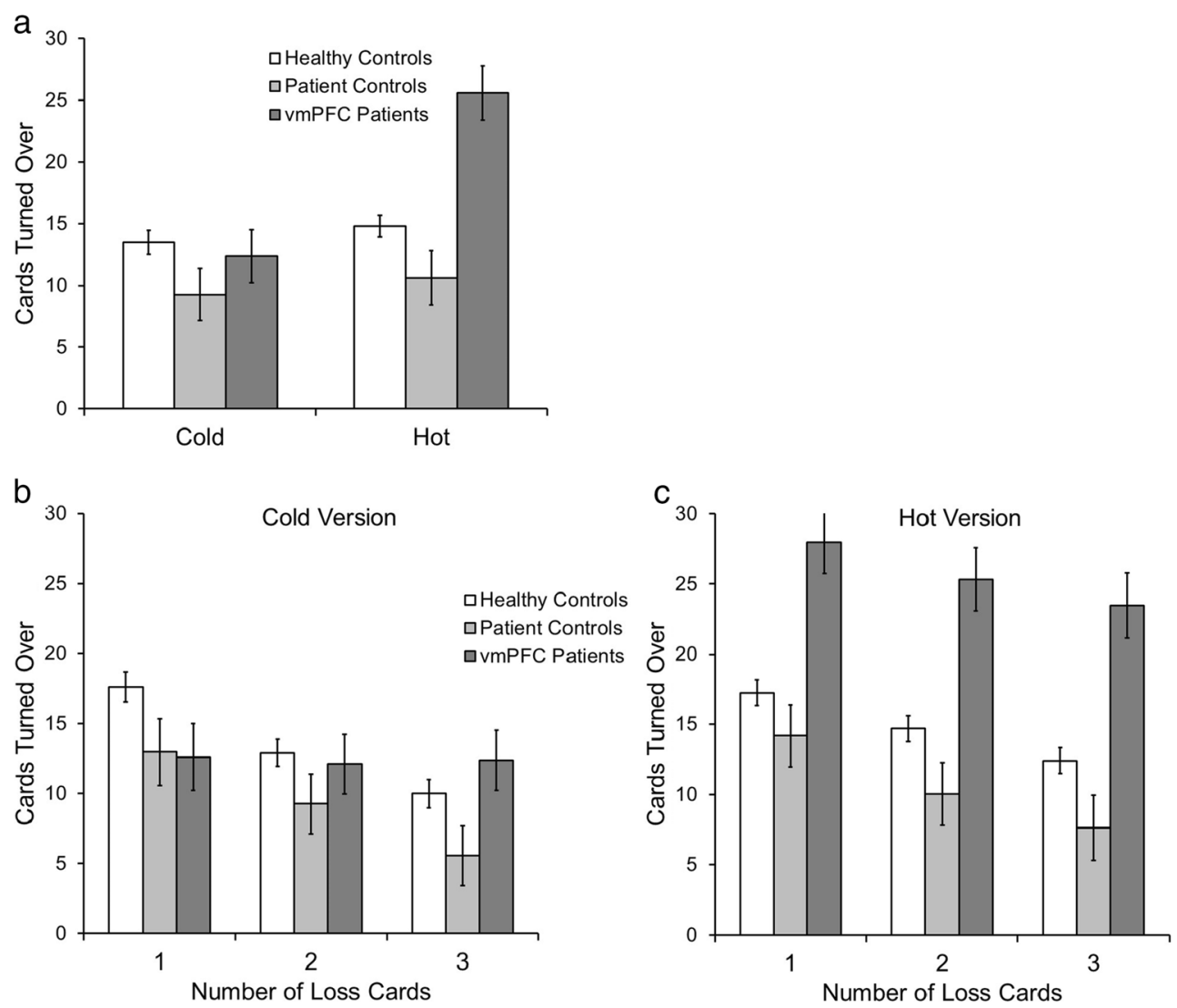

Fig. 3 Risk taking, defined as the number of cards turned over, as a function of participant group and experimental conditions. Panel A: Increase in risk appetite among vmPFC patients, relative to both other participant groups, in the hot CCT only. Panel B: Insensitivity to trial-

level variation in the number of loss cards (i.e., loss probability) in the vmPFC group in the cold CCT. Panel C: Sensitivity to trial-level variation in the number of loss cards in all three groups in the hot CCT. Error bars show the standard error of the mean 
however, the version effect was significant, $z=2.20, p=.03$. Overall, the nonparametric tests confirmed the results of the parametric tests.

Risk adjustment Gain amount had no significant effect on risk taking, $F(2,78)=.41, p=.69, \eta_{\mathrm{p}}{ }^{2}=.01$, and there was no significant Group $\times$ Gain Amount interaction, $F(4,78)=1.66$, $p=.17, \eta_{\mathrm{p}}^{2}=.08$. Loss amount had a significant effect, $F(2$, $78)=10.86, p<.01, \eta_{\mathrm{p}}{ }^{2}=.22$. Further analysis of this effect revealed a significant linear effect of loss amount on risk taking, $F(1,39)=13.98, p<.01, \eta_{\mathrm{p}}{ }^{2}=.26$, such that larger loss amounts led to reduced risk taking. There was no significant quadratic effect of loss amount, $F(1,39)=.05, p=.01, \eta_{\mathrm{p}}{ }^{2}<$ .01 , and the Group $\times$ Loss Amount interaction was not significant, $F(4,78)=1.69, p=.16, \eta_{\mathrm{p}}{ }^{2}=.08$. The main effect of loss probability was also significant, $F(2,78)=87.77, p<.01$, $\eta_{\mathrm{p}}{ }^{2}=.69$. It was further qualified by a Group $\times$ Loss Probability interaction, $F(4,78)=5.62, p<.01, \eta_{\mathrm{p}}{ }^{2}=.22$, and by a Group $\times$ Version $\times$ Loss Probability interaction, $F(4$, 39) $=7.53, p<.01, \eta_{\mathrm{p}}{ }^{2}=.28$.

To unpack the three-way interaction, we conducted followup ANOVAs separately for the hot and cold versions. In the hot version, there was a significant effect of loss probability, $F(2,78)=65.92, p<.01, \eta_{\mathrm{p}}{ }^{2}=.63$, but no significant Group $\times$ Loss Probability interaction, $F(2,78)=.43, p=.43, \eta_{\mathrm{p}}{ }^{2}=.05$. In the cold version, there was a significant effect of loss probability, $F(2,78)=46.80, p<.01, \eta_{\mathrm{p}}{ }^{2}=.55$, and a significant Group $\times$ Loss Probability Interaction, $F(4,78)=10.47, p<$ $.01, \eta_{\mathrm{p}}{ }^{2}=.35$. Healthy controls and patient controls reduced their risk taking as the probability of losing increased, $p \mathrm{~s}<.01$, whereas vmPFC patients did not rely on loss probability to modulate their risk taking, $p=.91$. However, when testing for group effects at each level of loss probability, no significant differences emerged, although there was a marginal effect at the lowest risk level (one loss card), $F(2,39)=3.03, p=.06$, $\eta_{\mathrm{p}}{ }^{2}=.13$. When we followed up this marginal effect with Bonferroni comparisons, no significant pairwise differences among the groups emerged, $p>.18$. The interaction of group, version, and loss probability is illustrated in Fig. 3 (bottom panels).

Again we also followed up the significant Group $\times$ Loss Probability interaction with nonparametric tests. Using a series of Friedman tests on mean ranks to capture effects of loss probability within each group, we found that control participants reduced their risk taking as the probability of losing increased (healthy controls: $\chi^{2}[2]=52.27, p<.01$; patient controls: $\chi^{2}[2]=10.33, p<.01$ ), whereas vmPFC patients did not rely on loss probability to modulate their risk taking, $\chi^{2}(2)=.09, p=.96$. However, when testing for group effects at each level of loss probability using Kruskal-Wallis tests, no significant differences emerged, $\chi^{2}(2) \leq 5.43, p \geq 0.07$. The results of the nonparametric tests thus aligned with those of the parametric tests.
Could the order of administration of the hot and cold CCT, which was counterbalanced across participants, have affected risk taking? To examine this possibility, we re-ran the ANOVA with task order (cold CCT first vs. hot CCT first) included as a between-subjects factor. There was no significant main effect of task order, nor any significant two-way interactions with group or version, on the number of cards drawn, $p>.05$. These findings suggest that task order did not affect overall risk appetite. Task order also showed no significant interactions with loss amount or with loss probability. There was, however, a significant interaction of task order and gain amount, $F(1.69,72)=5.34, p=.01, \eta_{p}{ }^{2}=$ .129. Follow-up ANOVAs, conducted separately within each task-order group, revealed no significant effects of gain amount, in either task-order group, $p>.05$. Overall, it does not appear that the pattern of results was influenced by the order in which participants encountered the hot and cold versions of the CCT.

Number of losses in the hot CCT In light of the finding that the vmPFC group showed increased risk appetite in the hot version, we analyzed the number of losses experienced in the hot CCT, collapsing across the 54 experimental trials and 12 dummy trials. A Kruskal-Wallis test showed that there was a statistically significant group difference in the number of losses, $\chi^{2}(2)=11.20, p<0.01$. Pairwise follow-up comparisons using the Mann-Whitney procedure revealed that the number of losses experienced by healthy controls $(M=$ 11.00; $S D=1.34)$ and by patient controls $(M=8.83 ; S D=$ 3.06) was not significantly different, $U=51.5, z=1.74, p=$ .08 . However, vmPFC patients $(M=18.50 ; S D=8.69)$ experienced more losses than healthy controls, $U=26.00, z=$ $2.86, p<.01$, and patient controls, $U=3.00, z=2.42, p=.02$. Thus, compared with both control groups, vmPFC patients showed greater risk appetite in the hot CCT despite more frequent loss feedback.

Final points score Did the group differences in risk appetite affect task success as measured by the final points score? A Kruskal-Wallis test showed no statistically significant group difference in the final points score in the cold CCT, $\chi^{2}(2)=$ $4.82, p=.09$. However, a significant group effect was present in the hot CCT, $\chi^{2}(2)=9.65, p<.01$, which we further probed with pairwise Mann-Whitney tests. Healthy controls $(M=$ 11,297.67; $S D=4,547.14)$ and patient controls $(M=$ 8,195.00; $S D=4,447.12$ ) showed no significant difference, $U=52.50, z=1.59, p=.11$, but final point scores of vmPFC patients $(M=18,851.67$; $\mathrm{SD}=6,188.08)$ were significantly higher than those of healthy controls, $U=27.00, z=2.67, p<$ .01 , and patient controls, $U=4.00, z=2.24, p=.03$. Thus, vmPFC patients' heightened risk appetite in the hot CCT resulted in a higher final points score, compared with both control groups. 
Response time A final follow-up question was whether group differences in risk appetite were associated with group differences in response time (RT). Such differences could shed light on the impulsivity with which participants made their selections. In the cold CCT, response time refers to the time between the onset of the choice array and the selection of a number field (e.g., "17") via mouse click. Given the positive skew of RT distributions for self-paced responses, we submitted each participant's median RT in the cold CCT to the analysis. A Kruskal-Wallis test on mean ranks for median RT was nonsignificant, $\chi^{2}(2)=4.76, p=.09$. Across the groups, the mean median RT in the cold CCT was 5,801 ms $(S D=$ 4,494ms).

In the hot CCT, separate RTs were recorded for each selection within a trial. Here, RT referred to the time elapsed since the onset of the choice array (for the first selection) or to the time elapsed since the previous selection (all subsequent selections). A Kruskal-Wallis test on mean ranks for median RT was significant, $\chi^{2}(2)=10.07, p<.01$. Follow-up MannWhitney tests showed no significant difference between healthy controls $(M=1,276 \mathrm{~ms} ; S D=536 \mathrm{~ms})$ and vmPFC patients $(M=948 \mathrm{~ms}, S D=238 \mathrm{~ms}), U=51.00, z=1.66, p=$ .10. In contrast, patient controls $(M=1,832 \mathrm{~ms} ; S D=331 \mathrm{~ms})$ differed from both vmPFC patients, $U=0.00, z=2.88, p<$ .01 , and healthy controls, $U=32.00, z=2.46, p=.01$. In summary, there was no evidence for RT differences between vmPFC patients and healthy controls, whereas patient controls responded more slowly than participants in both other groups.

\section{Discussion}

Using a lesion approach, we examined the role of vmPFC in risky choice under "hot" and "cold" conditions. In the hot CCT, participants made incremental decisions by turning over cards one-by-one with immediate feedback, the risk of an adverse outcome increasing with each card turned over. In the cold CCT, participants made decisions in a single step, without feedback. As hypothesized, the impact of vmPFC damage on risk taking was more pronounced in the hot version, though a subtle deficit also emerged in the cold version.

\section{VmPFC damage increases risk appetite under hot conditions}

In the hot CCT, vmPFC damage was associated with significantly increased risk appetite, consistent with prior findings in studies that have used laboratory gambling tasks (Clark et al., 2008; Studer et al., 2015). Compared with healthy and braindamaged controls, vmPFC patients turned over ten additional cards per trial on average, thereby achieving a higher final point score.
The pattern among vmPFC patients in the hot CCT is unlikely to result from difficulties understanding the task rules. All participants passed a comprehension check before starting the task, and showed normal probabilistic reasoning as assessed with the Abbreviated Numeracy Scale (Weller et al., 2013). VmPFC patients were also matched to the control groups on measures of fluid intelligence and mood, dimensions that could potentially affect risk taking. Moreover, the decision-relevant parameters were continously displayed on the computer screen, minimizing working-memory demands, and the task featured real monetary payoffs that may be easier to conceptualize than the hypothetical gains and losses used in some other studies (e.g., Clark et al., 2008; Pujara et al., 2015). Indeed, vmPFC patients demonstrated normal risk adjustment in the hot CCT (see below for additional discussion), which shows that their behavior was sensitive to the task contingencies. It is unlikely that vmPFC patients in the current study could have considered themselves immune to losses because they experienced more losses than participants in the other groups. Finally, the RT analysis offered no evidence of heightened impulsivity among vmPFC patients. Most critically, vmPFC patients' increased risk appetite was unique to the hot CCT, and was absent in the cold CCT. This suggests that vmPFC plays a critical role specifically when incremental decisions are followed by immediate reward feedback, and when risks are dynamic rather than static. In the following, we highlight several hypotheses that could be more closely examined in future work.

According to one view, vmPFC is involved in the generation of affective responses to fluctuations in internal and external states (Fellows, 2011; Hare, Camerer, \& Rangel, 2009; Roy et al., 2012). This theory yielded the hypotheses that motivated the current study. The interpretation in terms of a role of $\mathrm{vmPFC}$ in the construction of affective meaning is supported by findings from studies using the Iowa Gambling task (e.g., Bechara et al., 1994). Over the course of the task, healthy controls, but not vmPFC patients, developed an anticipatory skin conductance response before selecting cards from high-loss decks. This physiological response, in turn, was predictive of participant's avoidance of disadvantageous decks over time. In the current study, vmPFC damage may have undermined the affective response to losses, or the construction of an integrated response to the conflicting signals associated with each small immediate reward and the dynamically increasing risk of a large loss. This hypothesis could be further tested by examining the effect of vmPFC damage on autonomic responses during the hot CCT (see also Figner et al., 2009, Exp. 4).

A related explanation involves the role of vmPFC in the monitoring of cumulative reward in dynamic risk-taking contexts. In the hot CCT, cumulative trial earnings were saliently displayed on the screen (see Fig. 1). A recent fMRI study with healthy participants showed that vmPFC activation tracked 
cumulative reward, and that increases in cumulative reward over successive risky decisions was associated with heightened risk aversion in healthy individuals (Juechems et al., 2017). In naturalistic environments, this context-dependent risk taking mitigates the risk of catastrophic losses once a minimum reward threshold has been surpassed. By this account, damage to vmPFC may impair the regulation of shifts from risk seeking to risk aversion as a function of current reward.

A third possibility is that vmPFC damage increases the sensitivity to immediate reward, giving rise to a self-control deficit. Studies of intertemporal decision making have shown that vmPFC patients show steeper temporal discounting functions (Peters \& D'Esposito, 2016; Sellitto, Ciaramelli, \& di Pellegrino, 2010; but see Fellows \& Farah, 2005, for a conflicting finding). Interestingly, the increase in temporal discounting among vmPFC patients appears to be limited to intertemporal decisions in which the smaller-sooner option is available immediately (now trials), without extending to choices in which the smaller-sooner option involves a delay (Peters \& D'Esposito, 2016). A similar self-control deficit may also affect value-based decisions under risk with immediate outcomes (as in the hot CCT), while sparing decisions that do not involve immediate temptations (as in the cold CCT).

In summary, the finding of increased risk appetite in vmPFC patients in the hot CCT is compatible with several interpretations. We note that all of these interpretations are broadly consistent with a prominent view of vmPFC, mainly supported by functional neuroimaging work (Hare et al., 2009; Kahnt, Heinzle, Park, \& Haynes, 2011; Lim, O’Doherty, \& Rangel, 2013), as being involved in the computation of the subjective value of choice options, based on the relative weighting and integration of multiple attributes into a common currency (Levy \& Glimcher, 2012; Padoa-Schioppa \& Cai, 2011). Consistent with this hypothesis, vmPFC damage has been found to alter how specific attributes of choice options (e.g., timing and magnitude of reward) are weighted, although the component processes of valuation critically reliant on vmPFC are just beginning to be specified (Vaidya \& Fellows, 2015; Vaidya, Sefranek, \& Fellows, 2017).

We propose that in the hot CCT, vmPFC damage undermined the integration of affective features of the decision context with other choice-relevant features. In contrast, vmPFC damage did not impair sensitivity to outcome magnitude and probability. A recent study also found that vmPFC is critical to allow affective content to influence subjective value, while sparing the influence of perceptual information (Vaidya et al., 2017). While we favor the view of vmPFC as critically involved in the construction and integration of affective responses, future studies should rule out the possibility that vmPFC plays a (non-affective) role in the regulation of risk preferences in sequential decisions, or in control processes needed to instantiate (rather than construct) subjective value and preference during choice.

\section{Mixed effects of vmPFC damage on risk adjustment}

In the hot CCT, all three participant groups showed a similar reduction in risk taking with increasing loss amounts and loss probabilities. In contrast, the cold CCT revealed a group difference, with vmPFC patients (but not patient controls or healthy controls) failing to engage in risk adjustment in response to trial-level variation in loss probability. This finding is inconsistent with our prediction of abnormal risk taking among vmPFC patients under hot, rather than cold, conditions. It is possible that the monotonous nature of the cold CCT, in which participants made 66 sequential decisions without feedback, may have reduced vmPFC patients' monitoring of trial-varying decision parameters. The reason for the lack of modulation in vmPFC patients' behavior depending on loss probability in the cold CCT is currently not clear. It is worth noting, however, that vmPFC patients' choices were not significantly different from those of control participants, at any level of loss probability.

In contrast to prior studies with the CCT (e.g., Figner et al., 2009), gain amount had no effect on risk taking in the hot or the cold version, in any group. This may be due to the restricted range of gain amounts (10-30 points per gain card), particularly when compared to the range of loss amounts $(250,500$, or 750 points per loss card). In future studies, it may be useful to employ a broader range of gain amounts to avoid a floor effect of gain amount on risk taking.

Our mixed results on risk adjustment mirror heterogeneous findings in other studies, which have used a variety of riskychoice tasks. Previously-mentioned findings include intact risk adjustment in the Cambridge Gamble Task (Clark et al., 2008) and impaired risk adjustment in the Roulette Betting Task (Studer et al., 2015) and the Cups Task (Weller et al., 2007). However, these tasks were not designed to compare risk taking under hot and cold conditions. The Roulette Betting Task and the Cups Task (though not the Cambridge Gamble Task) have some hot aspects, such as real payouts. However, these tasks are also cold, in that they involve oneshot decisions without the dynamic, increasing-risk aspect of the hot CCT. Taken together, the current findings and those reported in the previous literature do not seem to warrant strong inferences about the necessity of vmPFC for risk adjustment.

\section{Limitations}

The current study had several limitations. First, the cold and hot versions of the CCT differ along multiple dimensions, including the presence of feedback, motor demands, the length of individual trials, and the static versus dynamic nature 
of the visual display. These differences are, to some extent, representative of real-world differences between hot and cold contexts (e.g., casino gambling, which presents increasingrisk dynamics in a complex environment, vs. investment planning, which presents static risks in an austere physical environment). Moreover, it is well-established that the hot and cold CCT dissociate with respect to their impact on physiological arousal as well as their sensitivity to developmental differences (e.g., Figner et al., 2009; Figner \& Weber, 2011). However, it is not clear which specific features of each task version are chiefly responsible for producing dissociations such as the one we observed in vmPFC patients. As a result, interactions between lesion status and CCT version could in principle result from features that are not centrally related to the cold-hot distinction (e.g., the increased motor demands of the hot CCT). Future studies could attempt to modify the classic CCT versions to reduce the potential influence of task confounds. Second, future lesion studies employing the CCT would benefit from the inclusion of physiological measures (e.g., pupil dilation, galvanic skin response), which would allow for a more direct test of the hypothesis that vmPFC damage alters affective responses in dynamic risk contexts.

Similar to other neuropsychological studies with patients with focal brain damage, the current study had a small sample size. This makes it important to replicate the study with larger, independent samples. Another issue is the inherent limitation in spatial resolution of human lesion studies. Brain lesions rarely respect anatomical boundaries and may encroach on regions adjacient to the one of interest, possibly contributing to the observed results. In this respect, we note that the lesions of vmPFC patients tested here involved in most cases the frontal pole, which has been consistently implicated in episodic future thinking by both neuroimaging (Benoit \& Schacter, 2015) and neuropsychological studies (Bertossi, Aleo, Braghittoni, \& Ciaramelli, 2016). Damage to this region may therefore have rendered vmPFC patients less capable than control patients to foresee the future consequences of their choices, leading them to focus instead on the promise of immediate gain.

\section{Conclusion}

The current study adds to the growing body of literature regarding the role of vmPFC in value-based decision making. It is the first study to show that affective context modulates the impact of vmPFC on risky choice. Under hot, affect-charged conditions, vmPFC damage was associated with increased risk appetite. Under cold, deliberative conditions, vmPFC patients showed normal risk appetite. This dissociation lends support to the view of vmPFC as a hub for the generation of affective meaning from dynamic internal and external states, possibly driving the optimal integration of affective feature of choice options with different categories of information into a subjective value that drives decision making and regulates risk taking.
Author Note We are grateful to acknowledge Davide Braghittoni for his help with patient recruitment, and Carson Pun for his assistance with computer programming.

\section{References}

Bechara, A., Damasio, A. R., Damasio, H., \& Anderson, S. W. (1994). Insensitivity to future consequences following damage to human prefrontal cortex. Cognition, 50, 7-15. https://doi.org/10.1016/ 0010-0277(94)90018-3

Bechara, A., Damasio, H., Tranel, D., \& Damasio, A. R. (1997). Deciding advantageously before knowing the advantageous strategy. Science, 275, 1293-1295. https://doi.org/10.1126/science.275.5304.1293

Bechara, A., Tranel, D., Damasio, H., \& Damasio, A. R. (1996). Failure to respond autonomically to anticipated future outcomes following damage to prefrontal cortex. Cerebral Cortex, 6, 215-225. https:// doi.org/10.1093/cercor/6.2.215

Benoit, R. G., \& Schacter, D. L. (2015). Specifying the core network supporting episodic simulation and episodic memory by activation likelihood estimation. Neuropsychologia, 75, 450-457. https://doi. org/10.1016/j.neuropsychologia.2015.06.034.

Bertossi, E., Aleo, F., Braghittoni, D., \& Ciaramelli, E. (2016). Stuck in the here and now: Construction of fictitious and future experiences following ventromedial prefrontal damage. Neuropsychologia, 81: 107-116.

Bertossi, E., \& Ciaramelli, E. (2016). Ventromedial prefrontal damage reduces mind-wandering and biases its temporal focus. Social Cognitive and Affective Neuroscience, 11, 1783-1791. https://doi. org/10.1093/scan/nsw099

Bertossi, E., Tesini, C., Cappelli, A., \& Ciaramelli, E. (2016). Ventromedial prefrontal damage causes a pervasive impairment of episodic memory and future thinking. Neuropsychologia, 90, 1224. https://doi.org/10.1016/j.neuropsychologia.2016.01.034

Clark, L., Bechara, A., Damasio, H., Aitken, M. R. F., Sahakian, B. J., \& Robbins, T. W. (2008). Differential effects of insular and ventromedial prefrontal cortex lesions on risky decision-making. Brain, 131, 1311-1322. https://doi.org/10.1093/brain/awn066

D'Argembeau, A. (2013). On the role of the ventromedial prefrontal cortex in self-processing: The valuation hypothesis. Frontiers in Human Neuroscience, 7. https://doi.org/10.3389/fnhum.2013. 00372

Fellows, L. K. (2011). Orbitofrontal contributions to value-based decision making: Evidence from humans with frontal lobe damage. Annals of the New York Academy of Sciences, 1239, 51-58. https://doi.org/10. 1111/j.1749-6632.2011.06229.x

Fellows, L. K., \& Farah, M. J. (2005). Dissociable elements of human foresight: A role for the ventromedial frontal lobes in framing the future, but not in discounting future rewards. Neuropsychologia, 43, 1214-1221. https://doi.org/10.1016/j.neuropsychologia.2004.07. 018

Figner, B., Mackinlay, R. J., Wilkening, F., \& Weber, E. U. (2009). Affective and deliberative processes in risky choice: Age differences in risk taking in the Columbia Card Task. Journal of Experimental Psychology: Learning, Memory, and Cognition, 35, 709-730. https://doi.org/10.1037/a0014983

Figner, B., \& Weber, E. U. (2011). Who takes risks when and why? Current Directions in Psychological Science, 20, 211-216. https:// doi.org/10.1177/0963721411415790

Folstein, M. F., Folstein, S. E., McHugh, P. R., Roth, M., Shapiro, M. B., Post, F., ... Kiloh, L. G. (1975). Mini-mental state: A practical method for grading the cognitive state of patients for the clinician. Journal of Psychiatric Research, 12, 189-198. https://doi.org/10. 1016/0022-3956(75)90026-6 
Hare, T. A., Camerer, C. F., \& Rangel, A. (2009). Self-control in decisionmaking involves modulation of the vmPFC valuation system. Science, 324, 646-648. https://doi.org/10.1126/science.1168450

Henri-Bhargava, A., Simioni, A., \& Fellows, L. K. (2012). Ventromedial frontal lobe damage disrupts the accuracy, but not the speed, of value-based preference judgments. Neuropsychologia, 50, 15361542. https://doi.org/10.1016/j.neuropsychologia.2012.03.006

Holper, L., \& Murphy, R. O. (2014). Hemodynamic and affective correlates assessed during performance on the Columbia Card Task (CCT). Brain Imaging and Behavior, 8, 517-530. https://doi.org/ 10.1007/s11682-013-9265-9

Juechems, K., Balaguer, J., Ruz, M., \& Summerfield, C. (2017). Ventromedial prefrontal cortex encodes a latent estimate of cumulative reward. Neuron, 93, 705-714.e4. https://doi.org/10.1016/j. neuron.2016.12.038

Kahneman, D., \& Tversky, A. (1979). Prospect theory: An analysis of decision under risk. Econometrica, 47, 263-292. https://doi.org/10. $2307 / 1914185$

Kahnt, T., Heinzle, J., Park, S. Q., \& Haynes, J. D. (2011). Decoding different roles for vmPFC and dlPFC in multi-attribute decision making. Neuroimage, 56, 709-715. https://doi.org/10.1016/j. neuroimage.2010.05.058

Levy, D. J., \& Glimcher, P. W. (2012). The root of all value: A neural common currency for choice. Current Opinion in Neurobiology. https://doi.org/10.1016/j.conb.2012.06.001

Lim, S. L., O’Doherty, J. P., \& Rangel, A. (2013). Stimulus value signals in ventromedial PFC reflect the integration of attribute value signals computed in fusiform gyrus and posterior superior temporal gyrus. Journal of Neuroscience, 33, 8729-8741. https://doi.org/10.1523/ JNEUROSCI.4809-12.2013

Metereau, E., \& Dreher, J. C. (2015). The medial orbitofrontal cortex encodes a general unsigned value signal during anticipation of both appetitive and aversive events. Cortex, 63, 42-54. https://doi.org/10. 1016/j.cortex.2014.08.012

Myers-Schulz, B., \& Koenigs, M. (2012). Functional anatomy of ventromedial prefrontal cortex: Implications for mood and anxiety disorders. Molecular Psychiatry, 17, 132-141. https://doi.org/10.1038/ mp. 2011.88

O’Doherty, J., Kringelbach, M. L., Rolls, E. T., Hornak, J., \& Andrews, C. (2001). Abstract reward and punishment representations in the human orbitofrontal cortex. Nature Neuroscience, 4, 95-102. https://doi.org/10.1038/82959

Padoa-Schioppa, C., \& Cai, X. (2011). The orbitofrontal cortex and the computation of subjective value: Consolidated concepts and new perspectives. Annals of the New York Academy of Sciences, 1239, 130-137. https://doi.org/10.1111/j.1749-6632.2011.06262.x

Panno, A., Lauriola, M., \& Figner, B. (2013). Emotion regulation and risk taking: Predicting risky choice in deliberative decision making. Cognition and Emotion, 27, 326-334. https://doi.org/10.1080/ 02699931.2012 .707642

Paulus, M. P. (2007). Decision-making dysfunctions in psychiatry Altered homeostatic processing? Science, 318, 602-606. doi: https://doi.org/10.1126/science.1142997

Peters, J., \& D'Esposito, M. (2016) Effects of Medial Orbitofrontal Cortex Lesions on Self-Control in Intertemporal Choice. Current Biology 26 (19):2625-2628

Pujara, M. S., Wolf, R. C., Baskaya, M. K., \& Koenigs, M. (2015). Ventromedial prefrontal cortex damage alters relative risk tolerance for prospective gains and losses. Neuropsychologia, 79, 70-75. doi: https://doi.org/10.1016/j.neuropsychologia.2015.10.026

Rangel, A., Camerer, C., \& Montague, P. R. (2008). Neuroeconomics: The neurobiology of value-based decision-making. Nature Reviews Neuroscience, 9, 545-556. doi: https://doi.org/10.1038/nrn2357

Reber, J., Feinstein, J. S., O’Doherty, J. P., Liljeholm, M., Adolphs, R., \& Tranel, D. (2017). Selective impairment of goal-directed decisionmaking following lesions to the human ventromedial prefrontal cortex. Brain, 140, 1743-1756. doi: https://doi.org/10.1093/brain/ awx 105

Rorden, C., \& Brett, M. (2000). Stereotaxic display of brain lesions. Behavioural Neurology, 12, 191-200. https://doi.org/10.1155/ 2000/421719

Roy, M., Shohamy, D., \& Wager, T. D. (2012). Ventromedial prefrontalsubcortical systems and the generation of affective meaning. Trends in Cognitive Sciences, 16, 147-156. https://doi.org/10.1016/j.tics. 2012.01.005

Sellitto, M., Ciaramelli, E., \& di Pellegrino, G. (2010). Myopic discounting of future rewards after medial orbitofrontal damage in humans. Journal of Neuroscience, 30, 16429-16436. https://doi. org/10.1523/jneurosci.2516-10.2010

Spinnler, H., \& Tognoni, G. (1987). Standardizzazione e taratura italiana di test neuropsicologici. The Italian Journal of Neurological Science, 6, 1-120.

Studer, B., \& Clark, L. (2011). Place your bets: Psychophysiological correlates of decision-making under risk. Cognitive, Affective, \& Behavioral Neuroscience, 11, 144-158. https://doi.org/10.3758/ s13415-011-0025-2

Studer, B., Manes, F., Humphreys, G., Robbins, T. W., \& Clark, L. (2015). Risk-sensitive decision-making in patients with posterior parietal and ventromedial prefrontal cortex injury. Cerebral Cortex, 25, 1-9. https://doi.org/10.1093/cercor/bht197

Terracciano, A., McCrae, R. R., \& Costa, P. T. (2003). Factorial and construct validity of the Italian Positive and Negative Affect Schedule (PANAS). European Journal of Psychological Assessment, 19, 131-141. https://doi.org/10.1027//1015-5759.19.2. 131

Vaidya, A. R., \& Fellows, L. K. (2015). Testing necessary regional frontal contributions to value assessment and fixation-based updating. Nature Communications, 6, 10120.

Vaidya, A. R., Sefranek, M., \& Fellows, L. K. (2017). Ventromedial frontal lobe damage alters how specific attributes are weighed in subjective valuation. Cerebral Cortex. Advance online publication. https://doi.org/10.1093/cercor/bhx246

Watson, D., Clark, L. a., \& Tellegen, A. (1988). Positive and negative affect schedule (PANAS). Journal of Personality and Social Psychology, 54, 1063-1070. https://doi.org/10.1037/t03592-000

Weller, J. A., Dieckmann, N. F., Tusler, M., Mertz, C. K., Burns, W. J., \& Peters, E. (2013). Development and testing of an abbreviated numeracy scale: A Rasch Analysis Approach. Journal of Behavioral Decision Making, 26, 198-212. https://doi.org/10.1002/bdm.1751

Weller, J. A., Levin, I. P., Shiv, B., \& Bechara, A. (2007). Neural correlates of adaptive decision making for risky gains and losses. Psychological Science, 18, 958-964. https://doi.org/10.1111/j. 1467-9280.2007.02009.x

Publisher's note Springer Nature remains neutral with regard to jurisdictional claims in published maps and institutional affiliations. 